\title{
Relative infectiousness of SARS-CoV-2 vaccine breakthrough infections, reinfections, and primary infections
}

Laith J. Abu-Raddad (1) 1,2,3,4凶 , Hiam Chemaitelly (10) 1,2,3, Houssein H. Ayoub (1) ${ }^{5}$, Patrick Tang (1) ${ }^{6}$, Peter Coyle (1) 7,8,9, Mohammad R. Hasan (1) 6, Hadi M. Yassine (1) 8,10, Fatiha M. Benslimane (10 8,10, Hebah A. Al-Khatib ${ }^{8,10}$, Zaina Al-Kanaani ${ }^{7}$, Einas Al-Kuwari ${ }^{7}$, Andrew Jeremijenko (1) ${ }^{7}$, Anvar Hassan Kaleeckal ${ }^{7}$, Ali Nizar Latif7, Riyazuddin Mohammad Shaik7 , Hanan F. Abdul-Rahim¹, Gheyath K. Nasrallah (1) 8,10, Mohamed Ghaith Al-Kuwari ${ }^{12}$, Adeel A. Butt (10 ${ }^{3,7}$, Hamad Eid Al-Romaihi ${ }^{13}$, Abdullatif Al-Khal ${ }^{7}$, Mohametabd H. Al-Thani' ${ }^{13} \&$ Roberto Bertollini'3

SARS-CoV-2 breakthrough infections in vaccinated individuals and in those who had a prior infection have been observed globally, but the transmission potential of these infections is unknown. The RT-qPCR cycle threshold $(\mathrm{Ct})$ value is inversely correlated with viral load and culturable virus. Here, we investigate differences in RT-qPCR Ct values across Qatar's national cohorts of primary infections, reinfections, BNT162b2 (Pfizer-BioNTech) breakthrough infections, and mRNA-1273 (Moderna) breakthrough infections. Our matched-cohort analyses of the randomly diagnosed infections show higher mean $\mathrm{Ct}$ value in all cohorts of breakthrough infections compared to the cohort of primary infections in unvaccinated individuals. The $\mathrm{Ct}$ value is 1.3 (95\% Cl: 0.9-1.8) cycles higher for BNT162b2 breakthrough infections, 3.2 ( $95 \% \mathrm{Cl}: 1.9-4.5$ ) cycles higher for mRNA-1273 breakthrough infections, and 4.0 (95\% Cl: 3.5-4.5) cycles higher for reinfections in unvaccinated individuals. Since Ct value correlates inversely with SARS-CoV-2 infectiousness, these differences imply that vaccine breakthrough infections and reinfections are less infectious than primary infections in unvaccinated individuals. Public health benefits of vaccination may have been underestimated, as COVID-19 vaccines not only protect against acquisition of infection, but also appear to protect against transmission of infection.

\footnotetext{
${ }^{1}$ Infectious Disease Epidemiology Group, Weill Cornell Medicine-Qatar, Cornell University, Doha, Qatar. ${ }^{2}$ World Health Organization Collaborating Centre for Disease Epidemiology Analytics on HIV/AIDS, Sexually Transmitted Infections, and Viral Hepatitis, Weill Cornell Medicine-Qatar, Cornell University, Qatar Foundation - Education City, Doha, Qatar. ${ }^{3}$ Department of Population Health Sciences, Weill Cornell Medicine, Cornell University, New York, NY, USA. ${ }^{4}$ Department of Public Health, College of Health Sciences, QU Health, Qatar University, Doha, Qatar. ${ }^{5}$ Mathematics Program, Department of Mathematics, Statistics, and Physics, College of Arts and Sciences, Qatar University, Doha, Qatar. ${ }^{6}$ Department of Pathology, Sidra Medicine, Doha, Qatar. ${ }^{7}$ Hamad Medical Corporation, Doha, Qatar. ${ }^{8}$ Biomedical Research Center, Member of QU Health, Qatar University, Doha, Qatar. ${ }^{9}$ Wellcome-Wolfson Institute for Experimental Medicine, Queens University, Belfast, UK. ${ }^{10}$ Department of Biomedical Science, College of Health Sciences, Member of QU Health, Qatar University, Doha, Qatar. ${ }^{11}$ College of Health Sciences, QU Health, Qatar University, Doha, Qatar. ${ }^{12}$ Primary Health Care Corporation, Doha, Qatar. ${ }^{13}$ Ministry

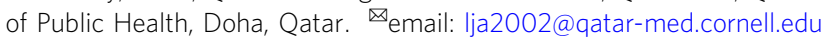


C oronavirus Disease 2019 (COVID-19) vaccines have demonstrated protection against the severe acute respiratory syndrome coronavirus 2 (SARS-CoV-2) ${ }^{1-3}$. However, the efficacy against acquisition of infection is imperfect in that vaccines have an efficacy $\mathrm{VE}_{\mathrm{S}}<100 \%$, particularly against variants of concern ${ }^{4-6}$. Breakthrough infections in vaccinated individuals have been documented ${ }^{4-9}$, but the transmission potential of these infections is poorly understood. It is conceivable that vaccinated persons who acquire the infection may be less infectious than unvaccinated persons who acquire the infection, as the vaccine-primed immune response may attenuate the natural history of infection, by reducing viral replication and accelerating viral clearance, leading to lower viral load and shorter duration of infection ${ }^{10,11}$. There is evidence that seems to support this hypothesis for SARS-CoV-2 infection ${ }^{12-14}$. Therefore, COVID-19 vaccines may be not only efficacious against acquisition of infection $\left(\mathrm{VE}_{\mathrm{S}}\right)$, but also against transmission of infection ${ }^{10,15,16}$, thereby adding an additional efficacy for each vaccine, denoted as $\mathrm{VE}_{\mathrm{I}}$ and defined as the proportional reduction in infectiousness among those infected but vaccinated, compared to those infected but unvaccinated ${ }^{10}$.

Leveraging the national, federated databases that have captured all SARS-CoV-2 vaccinations and polymerase chain reaction (PCR) testing in Qatar since the start of the epidemic ("Methods"), we investigated the effect of vaccination on infectiousness by comparing SARS-CoV-2 real-time (quantitative) reverse transcription-PCR (RT-qPCR) cycle threshold (Ct) values of individuals infected and fully vaccinated with the values of those infected and unvaccinated. The RT-qPCR Ct value is a measure of the inverse of viral load and correlates strongly with culturable virus; ${ }^{17}$ thus, it can be used as a proxy of SARS-CoV-2 infectiousness ${ }^{17-21}$. We also investigated the effect of prior infection on infectiousness at reinfection by comparing the RTqPCR Ct values among those reinfected with SARS-CoV-2 with the values among those with primary infection. For standardization of Ct values, the comparisons were conducted using only the RT-qPCR-confirmed infections diagnosed using the TaqPath COVID-19 Combo Kits (Thermo Fisher Scientific, USA ${ }^{22}$ ) which were used for $>85 \%$ of all RT-qPCR tests in Qatar ${ }^{4-6,22}$.

The comparisons were implemented utilizing: (i) the national cohort of all 384,452 RT-qPCR-confirmed primary infections since epidemic onset (February 28, 2020) until the end of the study (July 11, 2021; Fig. 1a); (ii) the national cohort of all 1695 RT-qPCR-confirmed reinfections during the same period (Fig. 1b); (iii) the national cohort of all 898,648 individuals vaccinated with the BNT162b2 ${ }^{1}$ (Pfizer-BioNTech) vaccine since the first recorded vaccination in Qatar on December 16, 2020 until the end of the study (July 11, 2021; Fig. 1c); and (iv) the national cohort of all 468,872 vaccinated individuals with the mRNA-1273 ${ }^{2}$ (Moderna) vaccine during the same period (Fig. 1d).

The BNT162b2 and mRNA-1273 vaccines have been the vaccines of choice in the national immunization campaign in Qatar ${ }^{4-6,8,9,23,24}$. In total, there has been 4777 breakthrough infections in those fully vaccinated with BNT162b2 $(0.61 \%$ of those fully vaccinated; Fig. 1c) and 306 mRNA-1273 breakthrough infections in those fully vaccinated with mRNA-1273 $(0.09 \%$ of those fully vaccinated; Fig. 1d). Mass immunization started with the BNT162b2 vaccine, and the mRNA-1273 vaccine was introduced only several weeks later. During this vaccination drive, Qatar experienced two back-to-back epidemic waves dominated by the Alpha ${ }^{25}$ (B.1.1.7) and Beta ${ }^{25}$ (B.1.351) variants, with the Beta wave contributing to most of the diagnosed infec-

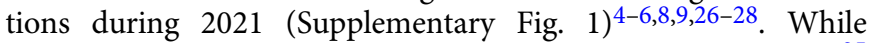
incidence since July 2021 has been dominated by the Delta ${ }^{25}$ (B.1.617.2) variant ${ }^{4-6,8,9,26-28}$, the number of Delta infections remained relatively low with no epidemic wave materializing as of the end date of this study, July 11, 2021.

Primary infection was defined as the first RT-qPCR-positive test for a given individual. Reinfection was defined as the first RTqPCR-positive test that occurred $\geq 90$ days after the primary infection $^{29-33}$. Breakthrough infection in a vaccinated individual was defined as an RT-qPCR-positive test 14 or more days after the individual received the second vaccine dose, conditional on this RT-qPCR-positive test being the first ever positive for this individual.

\section{Results}

Study populations. Table 1 shows the Ct value of the RT-qPCRpositive tests stratified by reason for testing for all SARS-CoV-2 infections diagnosed in Qatar using the TaqPath COVID-19 Combo Kits platform from February 28, 2020 to July 11, 2021. Among symptomatic infections, the mean Ct value was 23.1 (95\% CI: 23.0-23.1). Among asymptomatic infections, the mean $\mathrm{Ct}$ value was 25.2 (95\% CI: 25.1-25.2).

Figure 1 shows the process for identifying eligible primary infections, reinfections, BNT162b2 breakthrough infections, and mRNA-1273 breakthrough infections. Figure 2 schematizes the six pairwise comparisons conducted between these cohorts of infections, after exact matching to the first match in a 1:1 ratio by sex, 10-year age group, reason for RT-qPCR testing, and calendar week of the RT-qPCR test, to control for differences in biology by sex and age, as well as risk of exposure to SARS-CoV-2 in Qatar $34-38$ and variant exposure ${ }^{4-6,8,9,26-28}$ ("Methods"). Only the first RT-qPCR-positive test for each individual for each infection category was used for analysis.

Supplementary Tables 1 and 2 show demographic characteristics of the study populations (age, sex, and nationality) in the six pairwise comparisons. Scatter plots of the distribution of RTqPCR Ct values in each comparison were generated for all RTqPCR-confirmed infections, regardless of the reason for the RTqPCR testing (Supplementary Fig. 2), for only the randomly diagnosed (asymptomatic) infections (Fig. 3; "Methods"), and for only the symptomatic infections (Supplementary Fig. 3; "Methods").

Differences in RT-qPCR Ct values in all confirmed infections. In the comparisons including all RT-qPCR-confirmed infections, regardless of the reason for the RT-qPCR testing, the mean RTqPCR Ct value was higher in all cohorts of breakthrough infections compared to the cohort of primary infections in unvaccinated individuals (Table 2 and Supplementary Fig. 2). The Ct value was 1.0 (95\% confidence interval (CI): 0.7-1.2) cycles higher for BNT162b2 breakthrough infections, 3.5 (95\% CI: 2.5-4.5) cycles higher for mRNA-1273 breakthrough infections, and 3.8 (95\% CI: 3.4-4.2) cycles higher for reinfections in unvaccinated individuals. The Ct value was 3.1 (95\% CI: 2.0-4.2) cycles higher in mRNA-1273 breakthrough infections than in BNT162b2 breakthrough infections. Compared to reinfections in unvaccinated individuals, the $\mathrm{Ct}$ value was 2.1 (95\% CI: 0.33-3.8) cycles lower in mRNA-1273 breakthrough infections and 1.7 (95\% CI: 1.1-2.3) cycles lower in BNT162b2 breakthrough infections. All differences in Ct values were statistically significant with $p$ values $\leq 0.02$.

To account for the possibility of prolonged primary infections ${ }^{39-41}$, a comparison was restricted to reinfections in which the RT-qPCR-positive test occurred between 90 and 180 days after the primary infection. Compared to primary infections in unvaccinated individuals, the $\mathrm{Ct}$ value was $6.2(95 \%$ CI: 5.4-6.9) cycles higher for the reinfections in unvaccinated individuals ( $p$ values $\leq 0.001)$. 

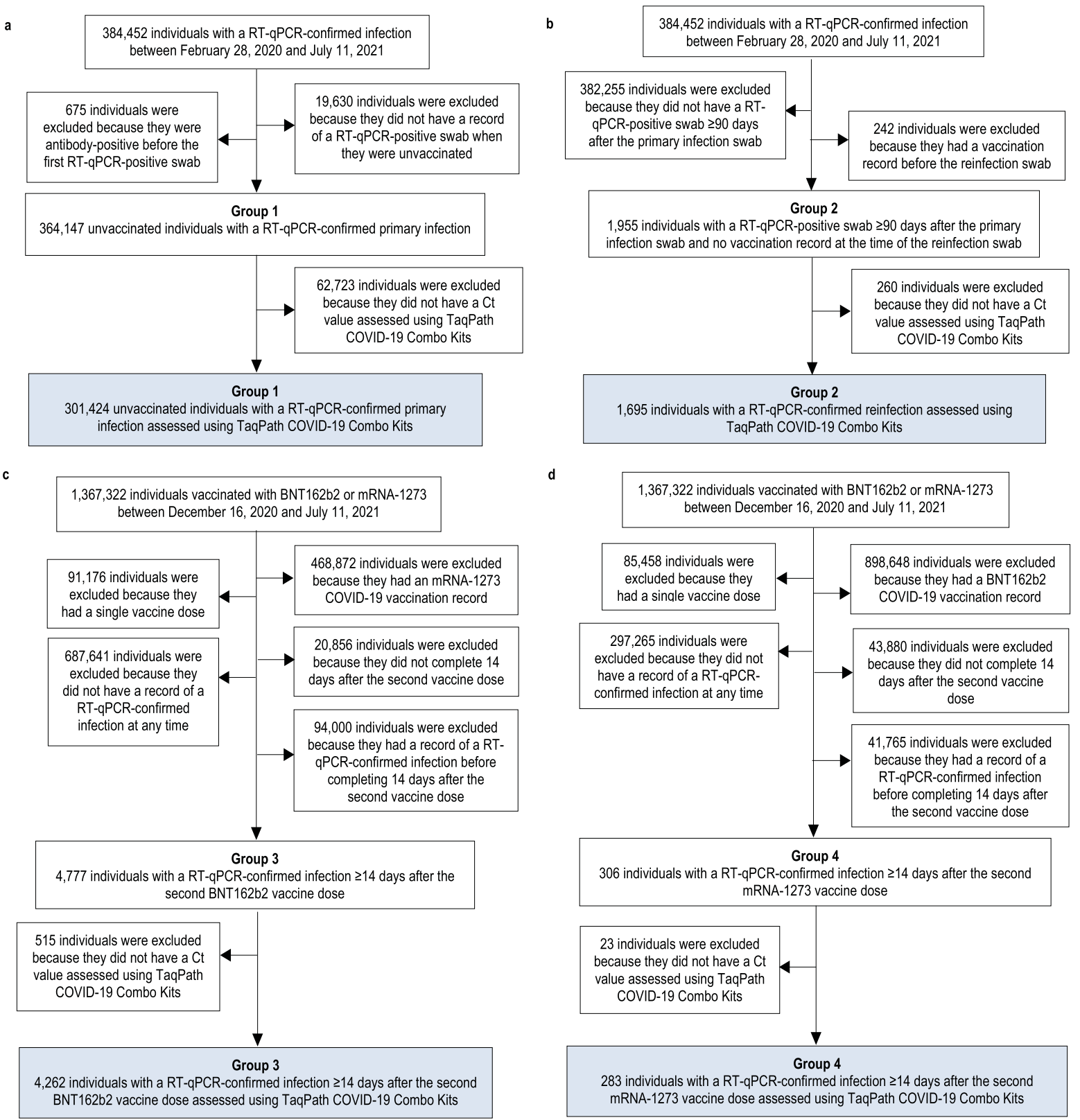

Abbreviations: $\mathrm{Ct}$, cycle threshold; RT-qPCR, real time reverse transcription polymerase chain reaction

Fig. 1 Flowchart illustrating the population selection process. Flowchart illustrating the selection of the cohorts of (a) primary infections in unvaccinated individuals, (b) reinfections in unvaccinated individuals, (c) BNT162b2-vaccine breakthrough infections, and (d) mRNA-1273-vaccine breakthrough infections.

The Ct values across the different infection categories were analyzed using matched pairwise comparisons, rather than using an overall regression model, for assessment of pairwise differences, improved statistical precision of the differences, and better control of confounding factors, considering the relatively small number of breakthrough infections in some of the categories. For additional validation of the results, all RTqPCR-confirmed infections were also analyzed using an overall regression model (Table 3). The results of this analysis were similar and consistent with the results of the main analysis using matched pairwise comparisons.

Differences in RT-qPCR Ct values in randomly diagnosed infections. In the comparisons including only the randomly diagnosed (asymptomatic) infections ("Methods"), which are perhaps most representative for differences between these cohorts of infections, given the random diagnosis, the mean RT-qPCR Ct value was also higher in all cohorts of breakthrough infections compared to the cohort of primary infections in unvaccinated individuals (Table 4 and Fig. 3). The Ct value was 1.3 (95\% CI: 0.9-1.8) cycles higher for BNT162b2 breakthrough infections, 3.2 (95\% CI: 1.9-4.5) cycles higher for mRNA-1273 breakthrough infections, and 4.0 (95\% CI: 3.5-4.5) cycles higher for reinfections in unvaccinated individuals. The Ct value was 2.2 (95\% CI: 0.9-3.6) cycles higher in mRNA-1273 breakthrough infections than in BNT162b2 breakthrough infections. Compared to reinfections in unvaccinated individuals, the $\mathrm{Ct}$ value was $2.0(95 \%$ CI: 1.2-2.8) cycles lower in BNT162b2 breakthrough infections and 1.7 (95\% CI: -0.3 to 3.7 ) cycles lower in mRNA-1273 breakthrough infections. All differences in Ct values were statistically significant with $p$ values $\leq 0.003$ except for the comparison 
Table 1 Characteristics of RT-qPCR Ct values in the study population.

\begin{tabular}{|c|c|c|c|c|c|c|c|}
\hline $\begin{array}{l}\text { Reason for RT-qPCR } \\
\text { testing }\end{array}$ & Setting of RT-qPCR testing & $\mathbf{N}$ & Proportion (\%) & Median & IQR & Mean $(95 \% \mathrm{Cl})$ & SD \\
\hline $\begin{array}{l}\text { Healthcare routine } \\
\text { testinga }\end{array}$ & Healthcare facility & 21,014 & 6.8 & 20.4 & $16.5-26.0$ & $21.5(21.4-21.6)$ & 6.2 \\
\hline Individual request & Healthcare facility & 15,489 & 5.0 & 24.3 & $19.1-30.2$ & $24.7(24.6-24.8)$ & 6.4 \\
\hline Contact tracing & $\begin{array}{l}\text { Healthcare facility \& } \\
\text { community }\end{array}$ & 67,159 & 21.8 & 25.0 & $19.9-30.3$ & $25.1(25.1-25.2)$ & 6.1 \\
\hline Port of entry ${ }^{a}$ & Healthcare facility & 33,287 & 10.8 & 28.5 & $21.0-32.1$ & $26.6(26.6-26.7)$ & 6.7 \\
\hline Other & $\begin{array}{l}\text { Healthcare facility \& } \\
\text { community }\end{array}$ & 6849 & 2.2 & 24.9 & $19.6-31.0$ & $25.3(25.1-25.4)$ & 6.4 \\
\hline
\end{tabular}

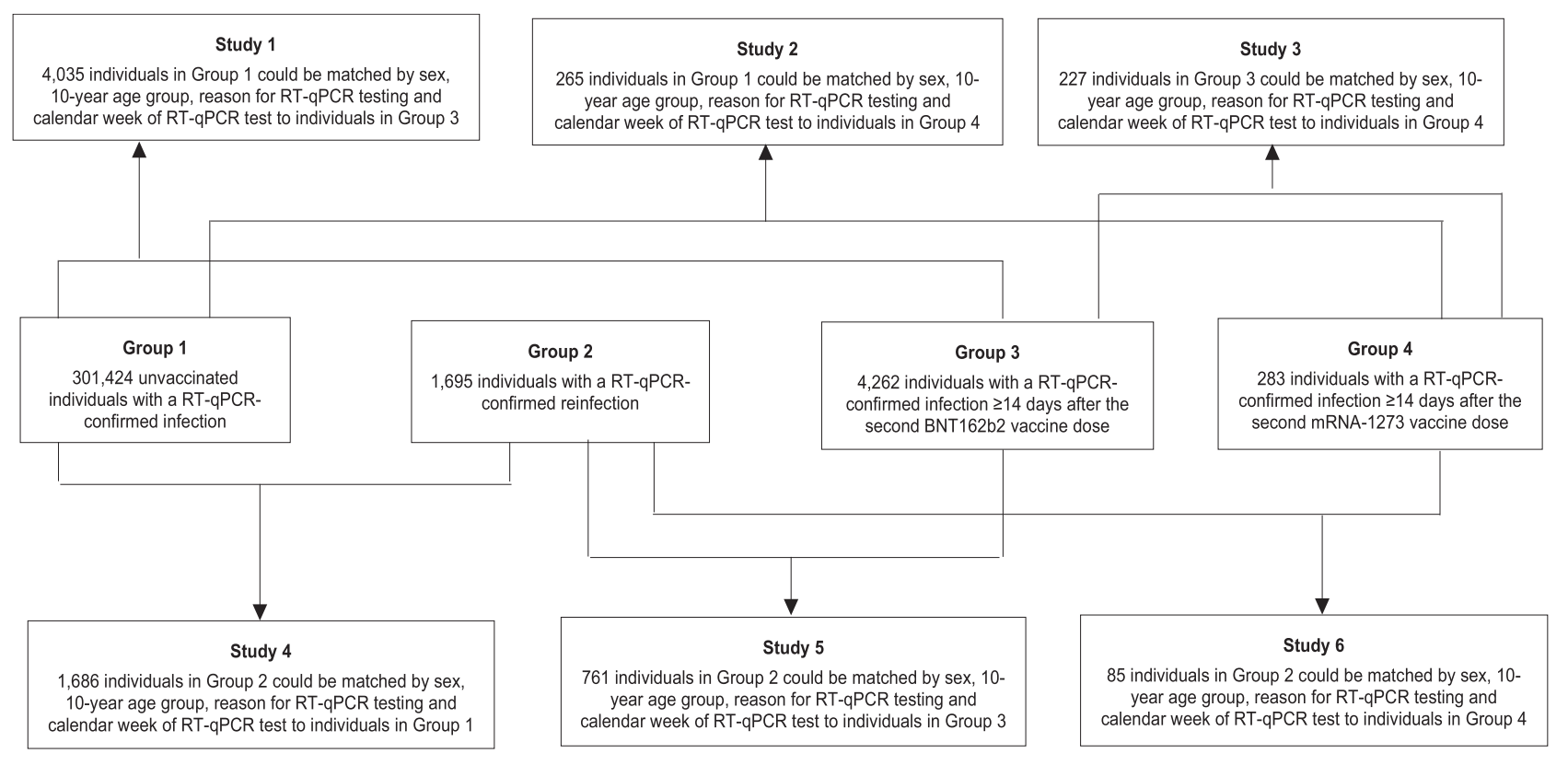

Abbreviations: RT-qPCR, real time reverse transcription polymerase chain reaction

Fig. 2 Schematic diagram showing the process of formulating the six pairwise comparisons between study cohorts. Schematic diagram showing the process of formulating the six pairwise comparisons between the cohorts of primary infections in unvaccinated individuals, reinfections in unvaccinated individuals, BNT162b2-vaccine breakthrough infections, and mRNA-1273-vaccine breakthrough infections, after 1:1 matching by sex, 10-year age group, reason for RT-qPCR testing, and calendar week of the RT-qPCR test.

between reinfections and mRNA-1273 breakthrough infections; likely a consequence of the small number of breakthrough infections that have been documented among those vaccinated with the mRNA-1273 vaccine in Qatar.

Differences in RT-qPCR Ct values in symptomatic infections. In the comparisons including only the symptomatic infections ("Methods"), the mean RT-qPCR Ct value was also higher in all cohorts of breakthrough infections compared to the cohort of primary infections in unvaccinated individuals, but the difference was smaller for BNT162b2 breakthrough infections (Table 5 and Supplementary Fig. 3). The Ct value was 0.2 (95\% CI: -0.2 to 0.6 ) cycles higher for BNT162b2 breakthrough infections, 4.9 (95\% CI: 2.3-7.4) cycles higher for mRNA-1273 breakthrough infections, and 3.8 (95\% CI: 2.9-4.7) cycles higher for reinfections in unvaccinated individuals. The $\mathrm{Ct}$ value was 5.3 (95\% CI: 2.6-8.1) cycles higher in mRNA-1273 breakthrough infections than in BNT162b2 breakthrough infections. Compared to reinfections in unvaccinated individuals, the $\mathrm{Ct}$ value was $1.8(95 \%$ CI: 0.6-3.0) cycles lower in BNT162b2 breakthrough infections and 6.4 (95\% CI: -0.04 to 12.9$)$ cycles lower in mRNA-1273 breakthrough infections. The differences in $\mathrm{Ct}$ values were generally statistically significant with $p$ values $\leq 0.05$ except for the comparison between primary infections and BNT162b2 breakthrough infections. Notably, very few symptomatic mRNA-1273 breakthrough infections have been documented in Qatar. This may have resulted in the borderline significance in the comparison between mRNA-1273 breakthrough infections and reinfections in unvaccinated individuals (Table 5). 

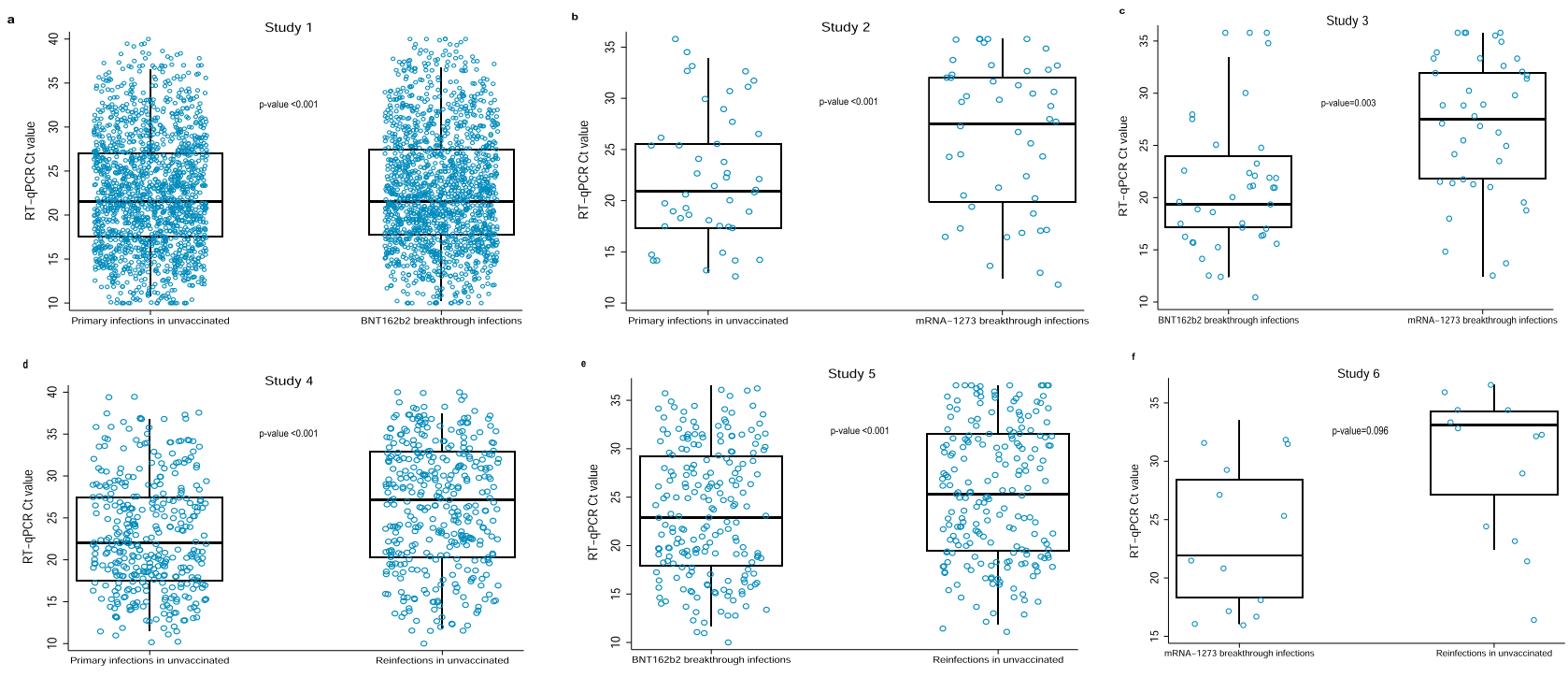

Fig. 3 RT-qPCR Ct values in the randomly diagnosed (asymptomatic) SARS-CoV-2 infections. Distribution of these Ct values (blue circles) in the six pairwise comparisons between primary infections in unvaccinated individuals, reinfections in unvaccinated individuals, BNT162b2-vaccine breakthrough infections, and mRNA-1273-vaccine breakthrough infections, a-f. A randomly diagnosed infection was defined as an RT-qPCR-positive test conducted with no prior reason to suspect infection and no reported presence of symptoms compatible with a respiratory tract infection. That is, the RT-qPCR test was conducted as part of a survey (random testing campaigns), for routine healthcare testing, for pre-travel requirement, or at port of entry upon arrival in Qatar. a includes, in each comparison group, $n=1584$ biologically independent samples, $\mathbf{b}$ includes $n=158$ biologically independent samples, $\mathbf{c}$ includes $n=140$ biologically independent samples, $\mathbf{d}$ includes $n=987$ biologically independent samples, e includes $n=421$ biologically independent samples, and f includes $n=60$ biologically independent samples, each over 1 experiment. Boxplots center lines indicate the median Ct values, box limits indicate the $25 \%$ and $75 \%$ quartiles, and whiskers indicate maximum and minimum observations within 1.5 of interquartile range. Paired $t$ tests were used to compare the difference in means between study groups, with no adjustment for multiple comparisons. Two-sided $p$-values are reported.

\section{Discussion}

Breakthrough infections in those vaccinated or who had a prior infection have higher RT-qPCR Ct values than primary infections in unvaccinated persons. While these breakthrough infections are not uncommon, the results indicate that they have lower viral load and are less likely to be infectious than primary infections. While some of these breakthrough infections could lead to secondary transmissions, and indeed some of them did have high viral loads (Fig. 3 and Supplementary Figs. 2 and 3), the risk of onward transmission is reduced, compared to primary infections. Thus, they are of less public health concern.

A consequence of these findings is that the public health benefits of vaccination may be underestimated. In addition to the conventional vaccine efficacy against acquisition of infection $\left(\mathrm{VE}_{\mathrm{S}}\right)$, that is assessed in randomized clinical trials ${ }^{1-3}$, there is an additional "breakthrough" efficacy against transmission ( $\mathrm{VE}_{\mathrm{I}}$ ) that augments the benefits of $\mathrm{VE}_{S}$, at least for the BNT162b2 and mRNA-1273 vaccines investigated in this study. The existence of this additional $\mathrm{VE}_{\mathrm{I}}$ efficacy implies that the reproduction number $\left(R_{0}\right)$ after vaccination is lower than current estimates ${ }^{10}$, and that SARS-CoV-2 incidence may decline faster with vaccine scale-up than previously thought ${ }^{10}$.

One finding of this study is that there appears to be a hierarchy in infectiousness of SARS-CoV-2 infections, where primary infections in unvaccinated persons are most infectious, followed by BNT162b2 breakthrough infections, mRNA-1273 breakthrough infections, and finally reinfections in unvaccinated persons. Strikingly, this hierarchy is the mirror image of the hierarchy observed in the efficacy against acquisition of infection. In our earlier studies on these national cohorts in Qatar, we found that those vaccinated with BNT162b2 had (relatively) the lowest protection against acquisition of infection (at 75\% against the Beta variant ${ }^{4,6,23,42}$ that dominated incidence since the onset of vaccination $\left.^{4-6,8,9,23,24,26-28}\right)$. Meanwhile, the protection against acquisition of infection was considerably higher among those vaccinated with mRNA-1273,6,42 and those with a prior infection $29-31,43$. This may indicate that both $\mathrm{VE}_{\mathrm{S}}$ and $\mathrm{VE}_{\mathrm{I}}$ are essentially inter-related manifestations of the strength of the vaccine-induced (or natural-infection-induced) immune response. When the immune response is strong against acquisition of infection, it also appears strong at reducing viral replication upon acquisition of the virus, leading to lower viral load and faster infection clearance. Thus, less secondary transmission occurs in breakthrough infections.

While it is well-established that the $\mathrm{Ct}$ value correlates inversely with SARS-CoV-2 infectiousness ${ }^{17-21}$, the mathematical form of the relationship between $\mathrm{Ct}$ value and transmission risk, or the mathematical form of the relationship between viral load and transmission risk, are inadequately understood ${ }^{17-21,44-47}$. If the transmission risk has a linear decreasing relationship with $\mathrm{Ct}$ value, as suggested by a nationwide study from Denmark ${ }^{18}$, the observed differences in $\mathrm{Ct}$ value by vaccination and prior infection status may not necessarily translate into large differences in transmission risk. However, if the transmission risk has a linear or a non-linear power-law relationship with viral load, as is the case for the rigorously established transmission risk of HIV infection $^{48-50}$, the observed differences in Ct value would translate into large differences in transmission risk.

This study has limitations. The number of documented mRNA-1273 breakthrough infections was small, thereby limiting the statistical precision of the comparisons involving these infections and leading to estimates with wider 95\% CIs; perhaps making them also more prone to bias. The matching further reduced the numbers of infections used in analysis and resulted in different subsets of each category of breakthrough infections being used in the different comparisons. With the relatively small 


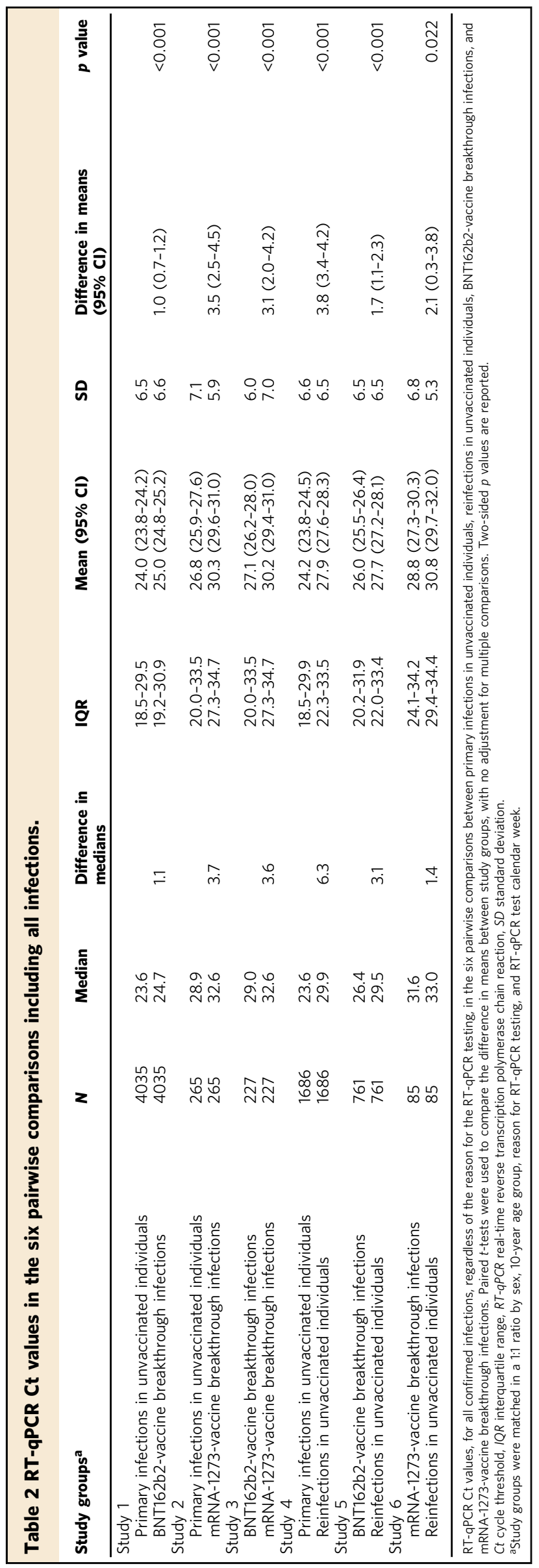

number of breakthrough infections, there was not adequate statistical power to investigate effect modifications by age or other demographic factors. With evidence for waning of vaccine immunity over time after the second dose $\mathrm{e}^{8,51-55}$, some of the differences observed for those vaccinated with BNT162b2 versus mRNA-1273 may be explained by the shorter duration between the second dose and the breakthrough infection for those mRNA1273 vaccinated, rather than differences in the biological immunity induced by each of these two vaccines. Future studies could investigate whether RT-qPCR Ct values are lower with increasing time since vaccination or secondary infection.

For symptomatic cases, the date of symptom onset was not available and thus an analysis of the duration between date of symptom onset and date of RT-qPCR testing, for the different categories of infections, was not possible. Only a small proportion of these documented infections was sequenced or RT-qPCR genotyped in Qatar; ${ }^{4-6,8,9,26-28}$ thus, we were unable to implement the above analyses for each variant separately. With the dominance of the Beta variant among cases included in this study (Supplementary Fig. 1), the results are most representative for this variant and may not be representative for the Alpha, Delta or other variants.

The study was implemented on documented infections, but other infections may have occurred and gone undocumented. It is possible that breakthrough infections in those vaccinated or who had a prior infection are less likely to be documented, perhaps because of minimal/mild or no symptoms. However, with the high rate of PCR testing in Qatar, the majority of infections are identified not because of testing symptomatic cases, but because of testing for other reasons, such as random testing campaigns, contact tracing, individual request, routine healthcare testing, pre-travel, and at ports of entry ${ }^{8}$. The results of our study were also consistent when we included only the randomly diagnosed infections.

Imperfect assay sensitivity and specificity of RT-qPCR testing may have affected infection ascertainment, but RT-qPCR testing was performed using a validated commercial platform that has been used globally and has essentially $100 \%$ sensitivity and specificity $^{22}$ ("Methods"). Unlike blinded, randomized clinical trials, the investigated observational cohorts were neither blinded nor randomized. Our cohorts predominantly included working-age adults; therefore, results may not necessarily be generalizable to other population groups, such as children or the elderly. Matching was done for sex, age, reason for the RTqPCR testing, and calendar week of the RT-qPCR test, but could not be done for other factors, such as comorbidities, as these were not available to study investigators. But inclusion of additional factors in the matching would have considerably reduced the sample sizes-breakthrough infections in those vaccinated or who had a prior infection are relatively uncommon. It is noteworthy that matching by age and sex may have served as a proxy for matching by co-morbidity, as comorbidities are associated with older age and may differ between females and males.

In conclusion, prior immunity, whether due to vaccination or prior infection, is associated with lower SARS-CoV-2 viral load upon infection. While breakthrough infections have been observed globally, they appear less infectious than primary infections; thus, they constitute a lesser public health concern. The public health benefits of vaccination are underestimated, as both the BNT162b2 and mRNA-1273 vaccines seem to protect not only against acquisition of infection, but also against transmission of infection. These findings justify optimism and stress the urgency to scale-up vaccination globally in order to robustly control infection transmission and the extent of the pandemic. 


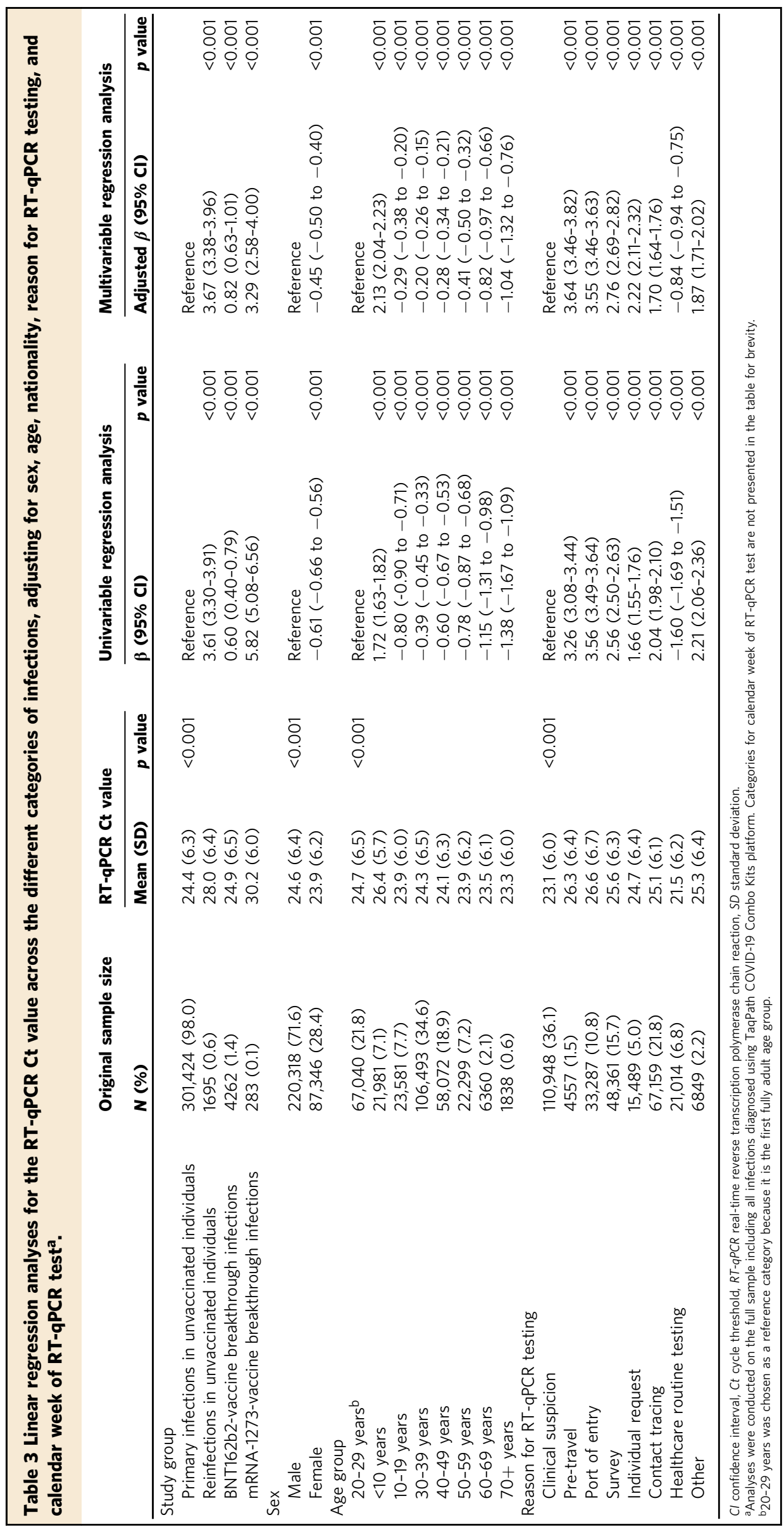




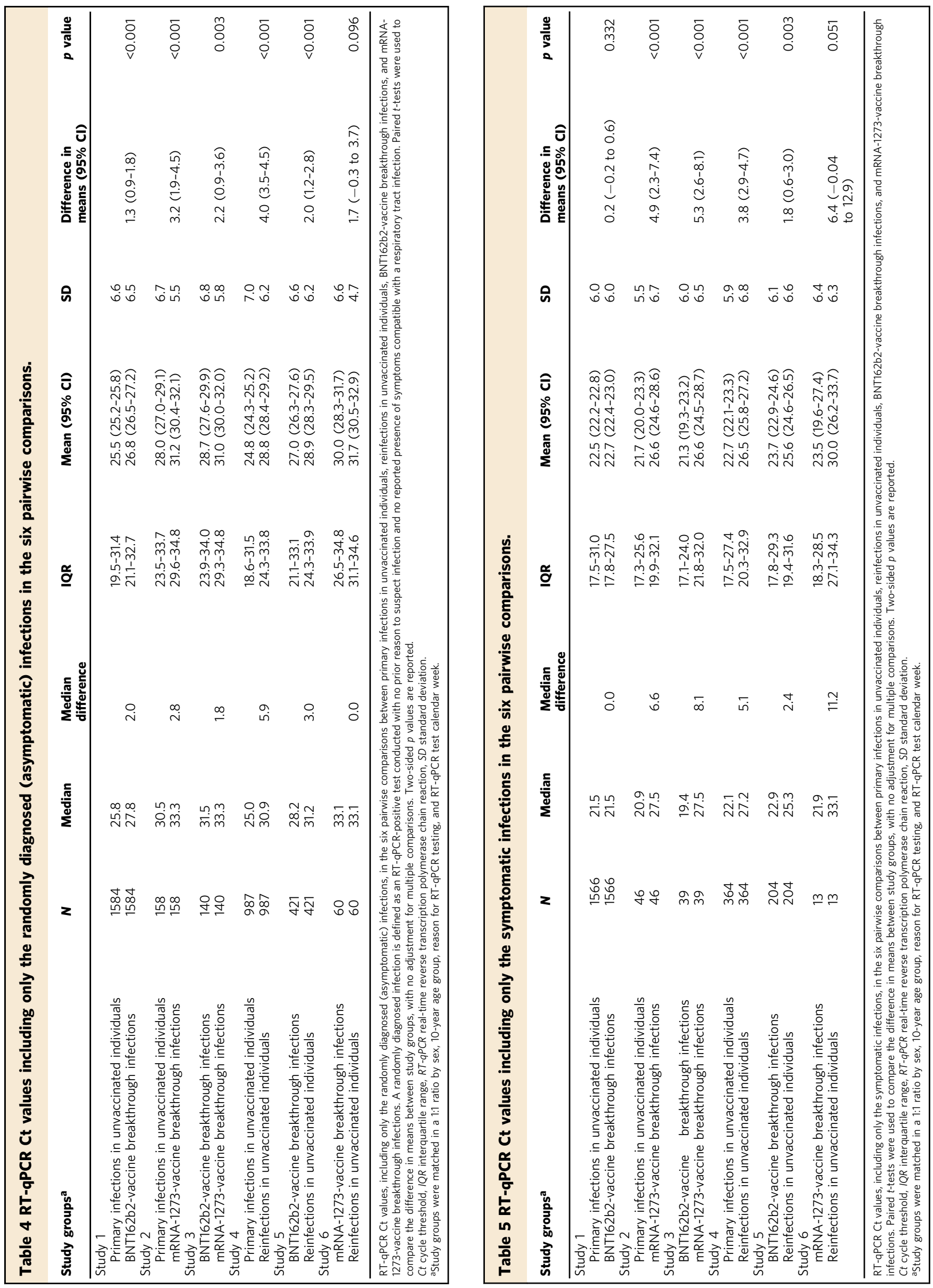




\section{Methods}

Data sources and study design. Analyses were conducted using the centralized, integrated, and standardized national severe acute respiratory syndrome coronavirus 2 (SARS-CoV-2) databases compiled at Hamad Medical Corporation (HMC), the main public healthcare provider and the nationally designated provider for all COVID-19 healthcare needs. Through a nationwide digital health information platform, these databases are complete, and have captured all SARSCoV-2-related data as well as related demographic details with no missing information since the start of the epidemic, including all records of PCR testing, antibody testing, COVID-19 hospitalizations, vaccinations, infection severity classification per World Health Organization (WHO) guidelines ${ }^{56}$ (performed by trained medical personnel through individual chart reviews), and COVID-19 deaths, also assessed per WHO guidelines ${ }^{57}$.

Every real-time reverse transcription-PCR (RT-qPCR) test conducted in Qatar, regardless of location (outpatient clinic, drive-thru, or hospital, etc.), is classified on the basis of symptoms and the reason for testing (clinical symptoms, contact tracing, random testing campaigns (surveys), individual requests, routine healthcare testing, pre-travel, and port of entry). Qatar has unique demographics by sex and nationality, since expatriates from over 150 countries comprise $89 \%$ of the population 34,58 .

The BNT162b2 and mRNA-1273 vaccines have been the vaccines of choice in the national immunization campaign in Qatar ${ }^{4-6,8,9,23,24}$. Nearly all individuals in the cohorts of this study were vaccinated (free of charge) in Qatar, rather than elsewhere. In rare situations where an individual received vaccination outside Qatar, that individual's vaccination details were still recorded in the health system at the port of entry upon return to Qatar, given the national requirements and to benefit from privileges associated with vaccination, such as quarantine exemption ${ }^{24}$.

Leveraging the national databases, effects of vaccination and of prior infection on SARS-CoV-2 infectiousness were investigated by comparing the RT-qPCR cycle threshold $(\mathrm{Ct})$ values in matched cohorts of primary infections in unvaccinated individuals, reinfections in unvaccinated individuals, BNT162b2 breakthrough infections, and mRNA-1273 breakthrough infections. Only the first RT-qPCRpositive test for each individual for each infection category was used in the analysis. These types of infections are defined in the main text.

In total, six types of pairwise comparisons were conducted on the cohorts of this study, after exact matching to the first match in a 1:1 ratio by sex, 10-year age group, reason for RT-qPCR testing, and calendar week of the RT-qPCR test, to control for differences in biology by sex and age, as well as exposure risk ${ }^{34-38}$ and variant exposure ${ }^{4-6,8,9,26-28}$. It is noteworthy that the first SARS-CoV-2 epidemic wave in Qatar occurred before introduction of any variant of concern and peaked in late May, 2020 34,35. The second wave was triggered by introduction and expansion of the Alpha ${ }^{25}$ (B.1.1.7) variant and peaked in early March, $2021^{4-6,8,9,26-28}$. The third wave was dominated by the $\operatorname{Beta}^{25}$ (B.1.351) variant, and peaked in the first week of April, 2021 $1^{4-6,8,9,26-28}$. The Delta ${ }^{25}$ (B.1.617.2) variant has been introduced only recently in Qatar, and as of July 11, 2021, it remains at low incidence ${ }^{4-6,8,9,26-28}$. There is no evidence that any other variant of concern is or has been responsible for appreciable community transmission in Qatar $4-6,8,9,26-28$

Comparisons across the cohorts of infection were implemented for all RTqPCR-confirmed infections, for only the symptomatic infections defined as RTqPCR-positive tests conducted because of clinical suspicion due to symptoms compatible with a respiratory tract infection, and for only the randomly diagnosed (asymptomatic) infections defined as RT-qPCR-positive tests conducted with no prior reason to suspect infection and no reported symptoms compatible with a respiratory tract infection. The latter was strictly defined as an RT-qPCR-positive test conducted as part of a survey (random testing campaigns), for routine healthcare testing, as a pre-travel requirement, or at a port of entry upon arrival in the country $4-6,34$

All records of RT-qPCR testing in Qatar were examined in this study, but only samples of matched cohorts were included in the analysis. Individuals with a record of a SARS-CoV-2 antibody-positive test before the first RT-qPCR-positive test were excluded from analysis of those with primary infections. Individuals with a record of vaccination before the reinfection diagnosis were excluded from the analysis of those with reinfection. Only breakthrough infections in fully vaccinated individuals were included in the analysis. Being fully vaccinated was defined as completion of $\geq 14$ days after the second dose. Only individuals who had their first and second doses with the same vaccine were included in the analysis.

Reporting of the study followed the STROBE guidelines (Supplementary Table 3).

Laboratory methods. Nasopharyngeal and/or oropharyngeal swabs were collected for PCR testing and placed in Universal Transport Medium (UTM). Aliquots of UTM were extracted on a QIAsymphony platform (QIAGEN, USA) and tested with RT-qPCR using TaqPath COVID-19 Combo Kits (100\% sensitivity and specificity; ${ }^{22}$ Thermo Fisher Scientific, USA) on an ABI 7500 FAST (Thermo Fisher, USA); extracted using a custom protocol ${ }^{59}$ on a Hamilton Microlab STAR (Hamilton, USA) and tested using AccuPower SARS-CoV-2 Real-Time RT-PCR Kits (100\% sensitivity and specificity; ${ }^{60}$ Bioneer, Korea) on an ABI 7500 FAST; or loaded directly into a Roche cobas 6800 system and assayed with a cobas SARS-
CoV-2 Test (95\% sensitivity, $100 \%$ specificity $^{61}$; Roche, Switzerland). The first assay targets the viral S, N, and ORFlab regions. The second targets the viral RdRp and E-gene regions, and the third targets the ORF1ab and E-gene regions.

For standardization of $\mathrm{Ct}$ values, only the RT-qPCR-confirmed infections diagnosed using the TaqPath COVID-19 Combo Kits platform (Thermo Fisher Scientific, $\mathrm{USA}^{22}$ ) were included in the analysis. This platform was used for $>85 \%$ of all RT-qPCR tests in Qatar and reports individual Ct values for each of the N ORF1ab, and $S$ genes ${ }^{4-6,22}$. The average of these three Ct values was included in the analysis. The correlation between each pair of these Ct values across all RT-qPCR positive tests was very strong with a Pearson correlation coefficient $\geq 0.976$. In the case of a gene "target failure", and specifically an S-gene "target failure" that was the defining characteristic of the Alpha cases ${ }^{62-64}$, the average was determined using only the two $\mathrm{Ct}$ values of the $\mathrm{N}$ and ORF1ab genes.

Antibodies against SARS-CoV-2 in serological samples were detected using a Roche Elecsys Anti-SARS-CoV-2 assay (99.5\% sensitivity ${ }^{65}, 99.8 \%$ specificity; ${ }^{65,66}$ Roche, Switzerland), an electrochemiluminescence immunoassay that uses a recombinant protein representing the nucleocapsid $(\mathrm{N})$ antigen for antibody binding. Results were interpreted according to the manufacturer's instructions (reactive: optical density (proxy for antibody titer ${ }^{67}$ ) cutoff index $\geq 1.0$ vs. nonreactive: optical density cutoff index $<1.0$ ).

All testing was conducted at the HMC Central Laboratory or Sidra Medicine Laboratory, following standardized protocols.

Statistical analysis. Socio-demographic characteristics of study samples were described using frequency distributions and measures of central tendency. Differences in proportions across categorical variables between study groups were evaluated using Chi-square tests. The distributions of the RT-qPCR Ct values were illustrated using scatter plots and boxplots, and summarized using measures of central tendency and dispersion. Mean differences in the RT-qPCR Ct values between study groups and associated 95\% CIs were calculated using paired $t$-tests. Two-sided $p$ value of $\leq 0.05$ indicated a significant association.

To provide an additional validation of the results, associations with a higher RT-qPCR Ct value across study groups and matching factors were explored using analysis of variance tests and univariable linear regressions. Beta coefficients ( $\beta \mathrm{s})$, 95\% CIs, and two-sided p-values were reported. All covariates were included in the multivariable analysis to estimate the adjusted $\beta$ s and associated $95 \%$ CIs and $p$ values. Covariates with $p$ value $\leq 0.05$ in the multivariable model were considered associated with the RT-qPCR Ct value.

Statistical analyses were conducted in STATA/SE version $17.0^{68}$.

Ethical approval. The study was approved by the Hamad Medical Corporation (MRC-05-011) and Weill Cornell Medicine-Qatar (20-00017) Institutional Review Boards with waiver of informed consent.

Reporting summary. Further information on research design is available in the Nature Research Reporting Summary linked to this article.

\section{Data availability}

The dataset of this study is a property of the Qatar Ministry of Public Health that was provided to the researchers through a restricted-access agreement that prevents sharing the dataset with a third party or publicly. The data are available under restricted access for confidentiality. Access can be obtained through a direct application for data access to Her Excellency the Minister of Public Health (https://www.moph.gov.qa/english/ OurServices/eservices/Pages/Governmental-Health-Communication-Center.aspx). The raw data are protected and are not available due to data privacy laws. Aggregate data are available within the manuscript and its Supplementary information.

\section{Code availability}

Standard epidemiological analyses were conducted using standard commands in STATA/ SE $17.0^{68}$. The commands/code are accessible using URL: https://github.com/ IDEGWCMQ/Ct-Infectiousness/blob/main/Code.do; https://doi.org/10.5281/ zenodo. $5751292^{69}$

Received: 27 July 2021; Accepted: 6 January 2022;

Published online: 27 January 2022

\section{References}

1. Polack, F. P. et al. Safety and efficacy of the BNT162b2 mRNA Covid-19 vaccine. N. Engl. J. Med. 383, 2603-2615 (2020).

2. Baden, L. R. et al. Efficacy and safety of the mRNA-1273 SARS-CoV-2 vaccine. N. Engl. J. Med. 384, 403-416 (2021).

3. Forni, G. \& Mantovani, A. Covid-19 Commission of Accademia Nazionale dei Lincei R. COVID-19 vaccines: where we stand and challenges ahead. Cell Death Differ 28, 626-639 (2021). 
4. Abu-Raddad, L. J., Chemaitelly, H. \& Butt, A. A., National Study Group for C-V. Effectiveness of the BNT162b2 Covid-19 vaccine against the B.1.1.7 and B.1.351 variants. N. Engl. J. Med. 385, 187-189 (2021).

5. Chemaitelly, $\mathrm{H}$. et al. mRNA-1273 COVID-19 vaccine effectiveness against the B.1.1.7 and B.1.351 variants and severe COVID-19 disease in Qatar. Nat. Med. 27, 1614-1621 (2021).

6. Tang, P. et al. BNT162b2 and mRNA-1273 COVID-19 vaccine effectiveness against the SARS-CoV-2 Delta variant in Qatar. Nat. Med. 27, 2136-2143 (2021).

7. CDC Covid-19-Vaccine Breakthrough Case Investigations Team. COVID-19 vaccine breakthrough infections reported to CDC - United States, January 1-April 30, 2021. Morb. Mortal. Wkly Rep. 70, 792-793 (2021).

8. Chemaitelly, H. et al. Waning of BNT162b2 Vaccine Protection against SARSCoV-2 Infection in Qatar. N. Engl. J. Med. 385, e83 (2021).

9. Abu-Raddad, L. J. et al. Association of prior SARS-CoV-2 infection with risk of breakthrough infection following mRNA vaccination in Qatar. JAMA 326, 1930-1939 (2021).

10. Makhoul, M. et al. Epidemiological impact of SARS-CoV-2 vaccination: mathematical modeling analyses. Vaccines 8, 668-684 (2020).

11. Singanayagam, A. et al. Community transmission and viral load kinetics of the SARS-CoV-2 delta (B.1.617.2) variant in vaccinated and unvaccinated individuals in the UK: a prospective, longitudinal, cohort study. Lancet Infect. Dis. S1473-3099, 00648-4 (2021).

12. Letizia, A. G. et al. SARS-CoV-2 seropositivity and subsequent infection risk in healthy young adults: a prospective cohort study. Lancet Respir. Med. $\mathbf{9}$, 712-720 (2021).

13. Thompson, M. G. et al. Prevention and attenuation of Covid-19 with the BNT162b2 and mRNA-1273 vaccines. N. Engl. J. Med. 385, 320-329 (2021).

14. Levine-Tiefenbrun, M. et al. Initial report of decreased SARS-CoV-2 viral load after inoculation with the BNT162b2 vaccine. Nat. Med. 27, 790-792 (2021).

15. Lipsitch, M. \& Kahn, R. Interpreting vaccine efficacy trial results for infection and transmission. Vaccine 39, 4082-4088 (2021).

16. Richterman, A., Meyerowitz, E. A. \& Cevik, M. Indirect protection by reducing transmission: ending the pandemic with SARS-CoV-2 vaccination. Open Forum Infect. Dis. ofab259, https://doi.org/10.1093/ofid/ofab259 (2021).

17. Singanayagam, A. et al. Duration of infectiousness and correlation with RTPCR cycle threshold values in cases of COVID-19, England, January to May 2020. Euro Surveill. 25, 2001483, https://doi.org/10.2807/15607917.ES.2020.25.32.2001483 (2020).

18. Lyngse, F. P. et al. Association between SARS-CoV-2 Transmissibility, Viral Load, and Age in Households. https://www.medrxiv.org/content/10.1101/ 2021.02.28.21252608v2 (2021).

19. Lee, L. Y. W. et al. SARS-CoV-2 infectivity by viral load, S gene variants and demographic factors and the utility of lateral flow devices to prevent transmission. Clin. Infect. Dis. ciab421, https://doi.org/10.1093/cid/ciab421 (2021).

20. Marks, M. et al. Transmission of COVID-19 in 282 clusters in Catalonia, Spain: a cohort study. Lancet Infect. Dis. 21, 629-636 (2021).

21. Bullard, J. et al. Predicting infectious severe acute respiratory syndrome coronavirus 2 from diagnostic samples. Clin. Infect. Dis. 71, 2663-2666 (2020).

22. Thermo Fisher Scientific. TaqPath COVID-19 CE-IVD RT-PCR Kit instructions for use (accessed 2 December 2020). https://assets.thermofisher.com/TFS-Assets/ LSG/manuals/MAN0019215_TaqPathCOVID-19_CE-IVD_RT-PCR\% 20Kit_IFU.pdf.

23. Abu-Raddad, L. J. et al. Pfizer-BioNTech mRNA BNT162b2 Covid-19 vaccine protection against variants of concern after one versus two doses. J. Travel Med. 28, taab083, https://doi.org/10.1093/jtm/taab083 (2021).

24. Bertollini, R. et al. Associations of vaccination and of prior infection with positive PCR test results for SARS-CoV-2 in airline passengers arriving in Qatar. JAMA 326, 185-188 (2021).

25. World Health Organization. Tracking SARS-CoV-2 variants (accessed 5 June 2021). https://www.who.int/en/activities/tracking-SARS-CoV-2-variants/.

26. National Project of Surveillance for Variants of Concern and Viral Genome Sequencing. Qatar viral genome sequencing data. Data on randomly collected samples. https://www.gisaid.org/phylodynamics/global/nextstrain/ (2021).

27. Benslimane, F. M. et al. One year of SARS-CoV-2: Genomic characterization of COVID-19 outbreak in Qatar. https://www.medrxiv.org/content/10.1101/ 2021.05.19.21257433v2 (2021).

28. Hasan, M. R. et al. Real-time SARS-CoV-2 Genotyping by High-Throughput Multiplex PCR reveals the epidemiology of the variants of concern in Qatar. Int. J. Infect. Dis. 112, 52-54 (2021).

29. Abu-Raddad, L. J. et al. SARS-CoV-2 antibody-positivity protects against reinfection for at least seven months with 95\% efficacy. EClinicalMedicine 35, 100861 (2021).

30. Abu-Raddad, L. J. et al. Assessment of the risk of severe acute respiratory syndrome coronavirus 2 (SARS-CoV-2) reinfection in an intense reexposure setting. Clin. Infect. Dis. 73, e1830-e1840 (2021).

31. Chemaitelly, H., Bertollini, R. \& Abu-Raddad, L. J. on behalf of the National Study Group for COVID-19 Epidemiology. Efficacy of natural immunity against SARS-CoV-2 reinfection with the Beta variant. N. Engl. J. Med. 385, 2585-2586 (2021)

32. Hansen, C. H., Michlmayr, D., Gubbels, S. M., Molbak, K. \& Ethelberg, S. Assessment of protection against reinfection with SARS-CoV-2 among 4 million PCR-tested individuals in Denmark in 2020: a population-level observational study. Lancet 397, 1204-1212 (2021).

33. Abu-Raddad, L. J., Chemaitelly, H. \& Bertollini, R. National Study Group for C-E. Severity of SARS-CoV-2 Reinfections as Compared with Primary Infections. N. Engl. J. Med. 385, 2487-2489 (2021).

34. Abu-Raddad, L. J. et al. Characterizing the Qatar advanced-phase SARS-CoV2 epidemic. Sci. Rep. 11, 6233 (2021).

35. Ayoub, H. H. et al. Mathematical modeling of the SARS-CoV-2 epidemic in Qatar and its impact on the national response to COVID-19. J. Glob. Health 11, 05005 (2021).

36. Coyle, P. V. et al. SARS-CoV-2 seroprevalence in the urban population of Qatar: an analysis of antibody testing on a sample of 112,941 individuals. iScience 24, 102646 (2021).

37. Al-Thani, M. H. et al. SARS-CoV-2 infection is at herd immunity in the majority segment of the population of Qatar. Open Forum Infect. Dis. 8, ofab221 (2021).

38. Jeremijenko, A. et al. Herd Immunity against severe acute respiratory syndrome coronavirus 2 infection in 10 communities, Qatar. Emerg. Infect. Dis. 27, 1343-1352 (2021)

39. Nussenblatt, V. et al. Year-long COVID-19 infection reveals within-host evolution of SARS-CoV-2 in a patient with B cell depletion. https:// www.medrxiv.org/content/10.1101/2021.10.02.21264267v2.full.pdf (2021)

40. Choi, B. et al. Persistence and evolution of SARS-CoV-2 in an immunocompromised host. N. Engl. J. Med. 383, 2291-2293 (2020).

41. Abu-Raddad, L. J. et al. Two prolonged viremic SARS-CoV-2 infections with conserved viral genome for two months. Infect. Genet. Evol. 88, 104684 (2021).

42. Abu-Raddad, L. J. et al. Protection offered by mRNA-1273 versus BNT162b2 vaccines against SARS-CoV-2 infection and severe COVID-19 in Qatar. https://www.medrxiv.org/content/10.1101/2021.11.12.21266250v1 (2021).

43. Abu-Raddad, L. J. et al. Introduction and expansion of the SARS-CoV-2 B.1.1.7 variant and its reinfections in Qatar: A nationally-representative cohort study. PLoS Med. 18, e1003879, https://doi.org/10.1371/journal.pmed.1003879 (2021).

44. La Scola, B. et al. Viral RNA load as determined by cell culture as a management tool for discharge of SARS-CoV-2 patients from infectious disease wards. Eur. J. Clin. Microbiol. Infect. Dis. 39, 1059-1061 (2020).

45. Platten, M. et al. SARS-CoV-2, CT-values, and infectivity-conclusions to be drawn from side observations. Viruses 13, 1459-1465 (2021).

46. Cevik, M. et al. SARS-CoV-2, SARS-CoV, and MERS-CoV viral load dynamics, duration of viral shedding, and infectiousness: a systematic review and meta-analysis. Lancet Microbe 2, e13-e22 (2021).

47. Tian, D. et al. Ct values do not predict severe acute respiratory syndrome coronavirus 2 (SARS-CoV-2) transmissibility in college students. J. Mol. Diagn. 23, 1078-1084 (2021).

48. Hughes, J. P. et al. Determinants of per-coital-act HIV-1 infectivity among African HIV-1-serodiscordant couples. J. Infect. Dis. 205, 358-365 (2012).

49. Quinn, T. C. et al. Viral load and heterosexual transmission of human immunodeficiency virus type 1. Rakai Project Study Group. N. Engl. J. Med. 342, 921-929 (2000).

50. Nagelkerke, N., Abu-Raddad, L. J., Awad, S. F., Black, V. \& Williams, B. A signature for biological heterogeneity in susceptibility to HIV infection? Infect. Dis. Model 3, 139-144 (2018).

51. Israel, A. et al. Elapsed time since BNT162b2 vaccine and risk of SARS-CoV-2 infection in a large cohort. https://www.medrxiv.org/content/10.1101/ 2021.08.03.21261496v1 (2021).

52. Thomas, S. J. et al. Six month safety and efficacy of the BNT162b2 mRNA COVID-19 vaccine. https://www.medrxiv.org/content/10.1101/ 2021.07.28.21261159v1 (2021)

53. Mizrahi, B. et al. Correlation of SARS-CoV-2-breakthrough infections to timefrom-vaccine. Nat. Commun. 12, 6379 (2021).

54. Pouwels, K. B. et al. Impact of Delta on viral burden and vaccine effectiveness against new SARS-CoV-2 infections in the UK. Nat. Med. 27, 2127-2135 (2021).

55. Keehner, J. et al. Resurgence of SARS-CoV-2 infection in a highly vaccinated health system workforce. N. Engl. J. Med. 385, 1330-1332 (2021).

56. World Health Organization. COVID-19 clinical management: living guidance (accessed 15 May 2021). https://www.who.int/publications/i/item/WHO2019-nCoV-clinical-2021-1.

57. World Health Organization. International guidelines for certification and classification (coding) of COVID-19 as cause of death (accessed 15 May 2021). https://www.who.int/classifications/icd/Guidelines_Cause_of_Death_COVID19-20200420-EN.pdf?ua $=1$.

58. Planning and Statistics Authority-State of Qatar. Qatar Monthly Statistics (accessed 26 May 2020). https://www.psa.gov.qa/en/pages/default.aspx. 
59. Kalikiri, M. K. R. et al. High-throughput extraction of SARS-CoV-2 RNA from nasopharyngeal swabs using solid-phase reverse immobilization beads. https://www.medrxiv.org/content/10.1101/2020.04.08.20055731v1 (2020).

60. Kubina, R. \& Dziedzic, A. Molecular and serological tests for COVID-19 a comparative review of SARS-CoV-2 coronavirus laboratory and point-of-care diagnostics. Diagnostics 10, 434-452 (2020).

61. US Food and Drug Administration. Cobas SARS-CoV-2: Qualitative assay for use on the Cobas 6800/8800 systems (accessed 2 December 2020). https:// www.fda.gov/media/136049/download.

62. European Centre for Disease Prevention and Control. Rapid increase of a SARS-CoV-2 variant with multiple spike protein mutations observed in the United Kingdom (accessed 10 February 2021). https://www.ecdc.europa.eu/ sites/default/files/documents/SARS-CoV-2-variant-multiple-spike-proteinmutations-United-Kingdom.pdf.

63. Galloway, S. E. et al. Emergence of SARS-CoV-2 B.1.1.7 Lineage - United States, December 29, 2020-January 12, 2021. Morb. Mortal. Wkly Rep. 70, 95-99 (2021)

64. Challen, R. et al. Risk of mortality in patients infected with SARS-CoV-2 variant of concern 202012/1: matched cohort study. BMJ 372, n579 (2021).

65. Muench, P. et al. Development and validation of the Elecsys anti-SARS-CoV-2 immunoassay as a highly specific tool for determining past exposure to SARSCoV-2. J. Clin. Microbiol. 58, e01694-20. https://doi.org/10.1128/JCM.0169420 (2020).

66. The Roche Group. Roche's COVID-19 antibody test receives FDA emergency use authorization and is available in markets accepting the CE mark (accessed 5 June 2020). https://www.roche.com/media/releases/med-cor-2020-0503.htm.

67. Oved, K. et al. Multi-center nationwide comparison of seven serology assays reveals a SARS-CoV-2 non-responding seronegative subpopulation. EClinicalMedicine 29, 100651 (2020).

68. StataCorp. Stata Statistical Software: Release 17. (StataCorp LLC, College Station, TX, 2021).

69. Chemaitelly, H. STATA code. https://github.com/IDEGWCMQ/CtInfectiousness/blob/main/Code.do; https://doi.org/10.5281/zenodo.5751292 (2021).

\section{Acknowledgements}

We acknowledge the many dedicated individuals at Hamad Medical Corporation, the Ministry of Public Health, the Primary Health Care Corporation, the Qatar Biobank, Sidra Medicine, and Weill Cornell Medicine-Qatar for their diligent efforts and contributions to make this study possible. The authors are grateful for support from the Biomedical Research Program and the Biostatistics, Epidemiology, and Biomathematics Research Core, both at Weill Cornell Medicine-Qatar, as well as for support provided by the Ministry of Public Health and Hamad Medical Corporation. The authors are also grateful for the Qatar Genome Programme for supporting the viral genome sequencing. The funders of the study had no role in study design, data collection, data analysis, data interpretation, or writing of the article. Statements made herein are solely the responsibility of the authors.

\section{Author contributions}

L.J.A. conceived and co-designed the study, led the statistical analyses, and co-wrote the first draft of the article. H.C. co-designed the study, performed the statistical analyses, and co-wrote the first draft of the article. All authors (L.J.A., H.C., H.H.A., P.T., P.C., M.R.H., H.M.Y., F.M.B., H.A.A., Z.A.K., E.A., A.J., A.H.K., A.N.L., R.M.S., H.F.A., G.K.N., M.G.A., A.A.B., H.E.A., A.A., M.H.A., R.B.) contributed to data collection and acquisition, database development, discussion and interpretation of the results, and to the writing of the manuscript, and have read and approved the final manuscript.

\section{Competing interests}

A.A.B. has received institutional grant funding from Gilead Sciences unrelated to the work presented in this paper. Otherwise, we declare no competing interests.

\section{Additional information}

Supplementary information The online version contains supplementary material available at https://doi.org/10.1038/s41467-022-28199-7.

Correspondence and requests for materials should be addressed to Laith J. Abu-Raddad.

Peer review information Nature Communications thanks Kåre Mølbak, Jared Bullard and the other, anonymous, reviewer(s) for their contribution to the peer review of this work.

Reprints and permission information is available at http://www.nature.com/reprints

Publisher's note Springer Nature remains neutral with regard to jurisdictional claims in published maps and institutional affiliations.

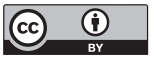

Open Access This article is licensed under a Creative Commons Attribution 4.0 International License, which permits use, sharing, adaptation, distribution and reproduction in any medium or format, as long as you give appropriate credit to the original author(s) and the source, provide a link to the Creative Commons license, and indicate if changes were made. The images or other third party material in this article are included in the article's Creative Commons license, unless indicated otherwise in a credit line to the material. If material is not included in the article's Creative Commons license and your intended use is not permitted by statutory regulation or exceeds the permitted use, you will need to obtain permission directly from the copyright holder. To view a copy of this license, visit http://creativecommons.org/ licenses/by/4.0/

(c) The Author(s) 2022 